\title{
Impacts of Temperature and Frequency on the Dielectric Properties for Insight into the Nature of the Charge Transports in the $\mathrm{Tl}_{2} \mathrm{~S}$ Layered Single Crystals
}

\author{
Aly M. Badr, Haroun A. Elshaikh, Ibraheim M. Ashraf \\ Physics Department, Faculty of Science, South Valley University, Aswan, Egypt \\ E-mail:badr_egsc@yahoo.com \\ Received August 24, 2010; revised September 25, 2010; accepted September 28, 2010
}

\begin{abstract}
Investigation of the electric properties of semi-conducting materials in an applied ac electric fields gives information about the nature of charge transport and localized states in the forbidden gap. Layered crystals usually contain structural defects, such as dislocations and vacancies that may form a high density of localized states near the Fermi level. So, the current study was carried out for insight into the dielectric Properties of $\mathrm{Tl}_{2} \mathrm{~S}$ layered single crystals. These properties were studied using the ac measurements in the low temperatures ranging from 77 to $300 \mathrm{~K}$. The real part of dielectric constant $\varepsilon^{\prime}$, imaginary part of dielectric constant $\varepsilon^{\prime \prime}$, the dissipation factor $\tan \delta$ and the alternating current conductivity $\sigma_{\mathrm{ac}}$ were measured in an applied ac electric field of frequencies extending from 2.5 to $50 \mathrm{kHz}$. Based on the dependencies of these dielectric parameters on both the frequency and temperature, the dielectric properties of the crystals under investigation were elucidated and analyzed. The ac conductivity was found to obey the power law $\sigma_{\mathrm{ac}}(\omega)=\mathrm{A} \omega^{\mathrm{s}}$ with which the values of the exponent $\mathrm{s}$ were evaluated to be less than unity in the range $0.21 \geq \mathrm{s} \geq 0.19$. Furthermore, it was found that the temperature dependence of ac conductivity follows the Arrhenius relation via which the impact of temperature on the electrical processes in an applied ac electric field was illustrated and analyzed. The influences of temperature and frequency on both the exponent $\mathrm{s}$ and band gap were also discussed in this investigation.
\end{abstract}

Keywords: Structural Defects in Layered Crystals, Dielectric Parameters, Nature of Charge Transports, Chalcogenide Semiconductors

\section{Introduction}

Due to difficulties in obtaining the $\mathrm{Tl}_{2} \mathrm{~S}$ compound in its single crystal forms, however, little is known about its properties. Synthetic $\mathrm{Tl}_{2} \mathrm{~S}$ is known to be a black, soft, and extremely laminar substance. In nature it was described as the rare mineral carlinite [1]. Earlier crystal structure work $[2,3]$ has shown that the atomic arrangement is similar to that of the anti-CdI 2 type, but with lattice parameters a and c tripled. A new description and a new discussion of the stereochemistry have been investigated by Giester et al. [4], in which they reported that the interatomic distances will always be given to two decimals $(\sigma<0.01 \AA)$, although for the $\mathrm{Tl}-\mathrm{Tl}$ distances three decimals would be adequate $(\sigma<0.005 \AA)$. In this study, there are two histograms that show a projection of the structure is parallel to the $\mathrm{x}_{1}$-axis and a projection of a single " $\mathrm{Tl}_{2} \mathrm{~S}$ sheet", which is parallel to the $\mathrm{z}$ - axis. Chalcogenide compounds can be considered as an important category of semi-conducting materials having extensive technical applications, and their single crystal forms are considered as a natural birefringence stratified medium $[5,6]$. The physical properties of layered crystals have constituted a major research area in solid-state physics. Layered crystals were largely used to test some of the most advanced techniques in modern photoemission [7]. This advantage has not been applied in large scale to chalcogenide semiconductors containing thallium [8]. The interest of these materials is stimulated not only by their fundamental properties but also by possible practical applications [9-14]. Layered crystals of chalcogenide semiconductors usually contain structural defects, such as dislocations and vacancies. The presence of these defects results in a high density of localized states near 
the Fermi level. So, it is interested to study of the electric properties of these materials in an applied ac electric field that can give information about the nature of charge transport between localized states in the forbidden gap [15]. It is reported in Refs. [16,17] that for the layered single crystals of chalcogenide semiconductors, the hopping conductivity in localized states may be occurred near the Fermi Level. Such measurements allow one to determine the relative dielectric constant $(\varepsilon)$, the loss factor $\left(\varepsilon^{\prime \prime}\right)$, the dissipation factor $(\tan \delta)$, and the alternating current conductivity $\left(\sigma_{\mathrm{ac}}\right)$. In order to execute the mechanism of charge transport, it is necessary to know the dependences of these parameters on frequency and temperature [15]. The dielectric behavior of materials under an external ac electric field has been the focus of numerous papers, in view of its high scientific and technological importance. These measurements were made in wide frequency and temperature ranges for many types of materials [18]. The aim of the current paper is mainly oriented to investigate of the frequency and temperature dependences of the dielectric parameters for $\mathrm{Tl}_{2} \mathrm{~S}$ layered single crystals and to clarify the mechanism of charge transport.

\section{Experimental Details and Conditions}

Layered single crystals of the $\mathrm{Tl}_{2} \mathrm{~S}$ semi-conducting compound was obtained via a new technique based on the Lenton Thermal Design (LTD), which is well described in Ref. [19]. The technique was provided with three digital temperature controllers (eurotherm 91e-type) that were used to control the temperature profile inside the tube furnace of this design. The temperature controllers were used to pinpoint a suitable fixed set inside the furnace at which a tube containing the $\mathrm{Tl}_{2} \mathrm{~S}$ compound was thereafter placed. The compound under investigation was obtained in mono-crystal form by cooling with a rate of $5^{\circ} \mathrm{C} / \mathrm{hr}$. The compound was prepared using starting materials of pure $(5 \mathrm{~N})$ elements that were evacuated $\left(10^{-6}\right.$ torr) in a silica tube of diameter $15 \mathrm{~mm}$. Natures of the obtained crystals were confirmed by comparing their recorded and standard X-ray diffraction patterns [20]. The comparison showed that the obtained compound is single crystal of $\mathrm{Tl}_{2} \mathrm{~S}$ with a rhombohedral (Hex) symmetry, space group: R3 (146) and lattice parameters: $a=$ 12.26 $\AA, c=18.29 \AA$, and $Z=27$. The obtained compound was obtained as layered single crystals from which the specimens were prepared by splitting the crystal along the cleavage plane and hence the resultant surfaces were mirror-like without any mechanical treatment. A High Precision LCR - 819 Meter $(12 \mathrm{~Hz}-100 \mathrm{kHz})$ was used for studying the dielectric (DE) properties of the $\mathrm{Tl}_{2} \mathrm{~S}$ layered single crystals. A piece of $1.2 \times 2.3 \mathrm{~mm}^{2}$ with a thickness of $0.6 \mathrm{~mm}$ is selected for the DE measurements. The contacts between the two opposite flat faces of the sample under study and the metal electrodes were painted using silver paste. The sample under investigation was mounted on the cold finger inside a cryostat (Oxford DN1704-type), which was evacuated to about $10^{-4}$ torr. Liquid nitrogen was used to maintain the temperature during the DE measurements and the temperature inside the cryostat was controlled by a digital temperature controller (Oxford ITC601-type).

\section{Results and Discussion}

Based on the dielectric measurements, two fundamental electrical characteristics of a material medium could be pinpointed: 1) The nature of the material as an insulating medium, which exhibits its capability to store electric charges; and 2) The nature of the material as a conductive medium, which exhibits its capability to transfer the electric charge. The Dielectric (DE) analysis is an important implement, which can be used to enrich knowledge with respect to the electrical properties of a material medium as a function of temperature and frequency. Furthermore, the DE analysis could be obtained for a material medium by carrying out the DE measurements. Based on the experimental data of these measurements, the DE parameters (e.g. the dielectric constant $\varepsilon^{\prime}$, dielectric $\operatorname{loss} \varepsilon^{\prime \prime}$, dissipation factor $\tan \delta$, and ac-C) of the material can be evaluated. In the current work, the frequency and temperature dependences of these parameters for the $\mathrm{Tl}_{2} \mathrm{~S}$ crystalline medium were described and analyzed as following: The complex dielectric constant of the material medium is generally formulated as two parts: $\varepsilon=\varepsilon^{\prime}+\mathrm{j} \varepsilon^{\prime \prime}$, where $\varepsilon^{\prime}$ is the real part of dielectric constant (RPDEC) and $\varepsilon "$ is the dielectric loss or the imaginary part of dielectric constant (IPDEC). Based on the relation between $\varepsilon^{\prime}$ and $\varepsilon^{\prime \prime}$, a loss tangent or the dissipation factor was defined as $\tan \delta=\varepsilon^{\prime \prime} / \varepsilon^{\prime}$. Dependences of the RPDEC, IPDEC, and dissipation factor $(\tan \delta)$ on both temperature and frequency of the applied ac field were individually investigated for $\mathrm{Tl}_{2} \mathrm{~S}$ layered single crystals. The DE measurements were carried out at the temperatures ranging from 77 to $300 \mathrm{~K}$ and frequencies ranging from 1 to $68.6 \mathrm{KHz}$. The aforementioned dielectric DE parameters were evaluated by measuring the equivalent parallel capacitance $C_{p}$ and the equivalent parallel resistance $R_{p}$ of the specimen under investigation using the following equations [21]:

$\varepsilon^{\prime}=C_{p} / C_{0} \Rightarrow \varepsilon^{\prime}=\left(\frac{C d}{\varepsilon_{0} A}\right)$, and $\varepsilon^{\prime \prime}=\left(\frac{\varepsilon^{\prime}}{\omega C_{p} R_{p}}\right)$

where $C_{p}$ is the capacitance of the specimen medium in Farad, $\mathrm{d}$ is the thickness in meter, $A$ is the area in square 
meter, $C_{0}$ is the geometrical capacitance of vacuum through the same dimensions as the sample, $\varepsilon_{0}$ is the absolute permittivity in the free space having a value of $8.854 \times 10^{-12} \mathrm{Fm}^{-1}, \varepsilon^{\prime}$ is the real part of dielectric constant and $\varepsilon^{\prime \prime}$ is the imaginary part of dielectric constant. Investigation of the electric properties of semiconductor materials in an applied ac electric fields gives information about the nature of charge transport and states in the forbidden gap [15]. So, the values of the alternating current conductivity (ac-C) for the $\mathrm{Tl}_{2} \mathrm{~S}$ crystalline medium were calculated based on the measured values of the aforementioned DE parameters using the following relation [22]:

$$
\sigma_{a c}(f)=2 \pi f \varepsilon_{0} \varepsilon^{\prime} \tan \delta
$$

where $f$ is the measuring frequency of the applied ac electric field $(\mathrm{Hz})$ and $\tan \delta$ is the dissipation factor that describes the phase difference between the current and voltage with respect to the applied ac electric field.

\subsection{Influences of Frequency and Temperature on $\varepsilon^{\prime}$}

Displacements of the charge carriers and hence the decrease of the RPDEC with increasing frequency are occurred in a dielectric material as a result of applying an ac electric field that is well known as polarization. The Fundamental interaction mechanisms between a dielectric material medium and electromagnetic waves are strongly required to be synopsized prior the insight into the discussion and analysis of the dielectric properties for the $\mathrm{Tl}_{2} \mathrm{~S}$ layered single crystals. The decrease of the dielectric constant with increasing frequency can be attributed to the contribution of many components of electrical polarization that are well specified as electronic, ionic (or atomic), dipolar (or orientation), and space charge [23, 24]: 1) Electronic polarization $\left(P_{e}\right)$ that arises due to the displacement of the valence electrons relative to the positive nucleus and occurs at the frequencies up to $10^{16}$ $\mathrm{Hz}$; 2) Ionic polarization $\left(P_{i}\right)$ that arises due to the displacement of negative and positive ions with respect to each other. In this category, the ions cannot be polarized rapidly because of they are heavier than electrons leading to that the maximum frequency of the ionic polarization is approximately $\left.10^{13} \mathrm{~Hz} ; 3\right)$ Dipolar polarization $\left(P_{d}\right)$ that arises due to the permanent electric dipole moments of molecules by which change orientation is governed into the direction of the applied electric field. Dipolar polarization occurs at frequencies up to about $10^{10} \mathrm{~Hz}$; and 4) Space charge polarization $\left(P_{s}\right)$ that arises due to the mobile charge carriers impeded by interfaces. Space charge polarization typically occurs at frequencies between 1 and $10^{3} \mathrm{~Hz}$.
Algebraic summation of all the aforementioned components for a dielectric material medium can be expressed as the total polarization $\mathrm{P}[23]$ :

$$
P=P_{e}+P_{i}+P_{d}+P_{s}
$$

The frequency dependencies of the electronic, ionic, dipolar and space charge polarization contributions to the relative dielectric constant are illustrated in the schematic diagram shown by Figure 1. Based on the bonding and structure of the dielectric material and the frequency, dielectric materials exhibit at least one of these polarization categories. The total polarization $\mathrm{P}$ is related to the relative dielectric constant $\varepsilon^{\prime}$ through the following equation [25]:

$$
P=\varepsilon_{0}\left(\varepsilon^{\prime}-1\right) E=\varepsilon_{0} \chi E
$$

where $\varepsilon_{0}$ is the permittivity of free space, $\chi$ is the electric susceptibility, and $\mathrm{E}$ is the applied ac electric field. Equation 4 shows the direct relation between relative dielectric constant and polarization. In accordance with this equation, one could predict the magnitude of the polarization in a dielectric material as the relative dielectric constant is well known for this material. The relative dielectric constant $\varepsilon^{\prime}$ determines the maximum energy that can be stored in the material. However, the relative loss factor $\varepsilon$ " evaluates the absorption of electrical energy by a dielectric material that is subjected to an alternating electromagnetic field. Furthermore, the dissipation factor $\tan \delta=\varepsilon^{\prime \prime} / \varepsilon^{\prime}$ determines how well a material can absorb the electromagnetic field [23,25]. The frequency dependence of the RPDEC for the $\mathrm{Tl}_{2} \mathrm{~S}$ layered single crystals was investigated in the frequency of the applied ac field ranging from $1-68.6 \mathrm{kHz}$ at different working temperatures extending from 77 to $300 \mathrm{~K}$. This variation of the RPDEC with frequency is shown in Figure 2. In accordance with this figure, a considerable decrease in the RPDEC commences from $1 \mathrm{kHz}$ till the frequency of $68.6 \mathrm{kHz}$ at all the working temperatures investigated here. It is evident from Figure 2 that the behavior of the $\mathrm{Tl}_{2} \mathrm{~S}$ crystalline medium could be interpreted with respect to the frequency intervals:

1) Behavior in the first interval is observed in the low frequency region $1-28.3 \mathrm{kHz}$ in which the RPDEC sharply decreases with increasing frequency. So, the RPDEC of the $\mathrm{Tl}_{2} \mathrm{~S}$ crystalline medium is therefore strongly dependent on the frequency at the low frequency region. The maximum value of the RPDEC is clearly observed at $1 \mathrm{kHz}$ for all the investigated working temperatures. However, the maximum value was found to be extended from 1982.58 to 3675.57 corresponding to the working temperatures ranging from 77 to $300 \mathrm{~K}$. So, the maximum value of the RPDEC at the working 


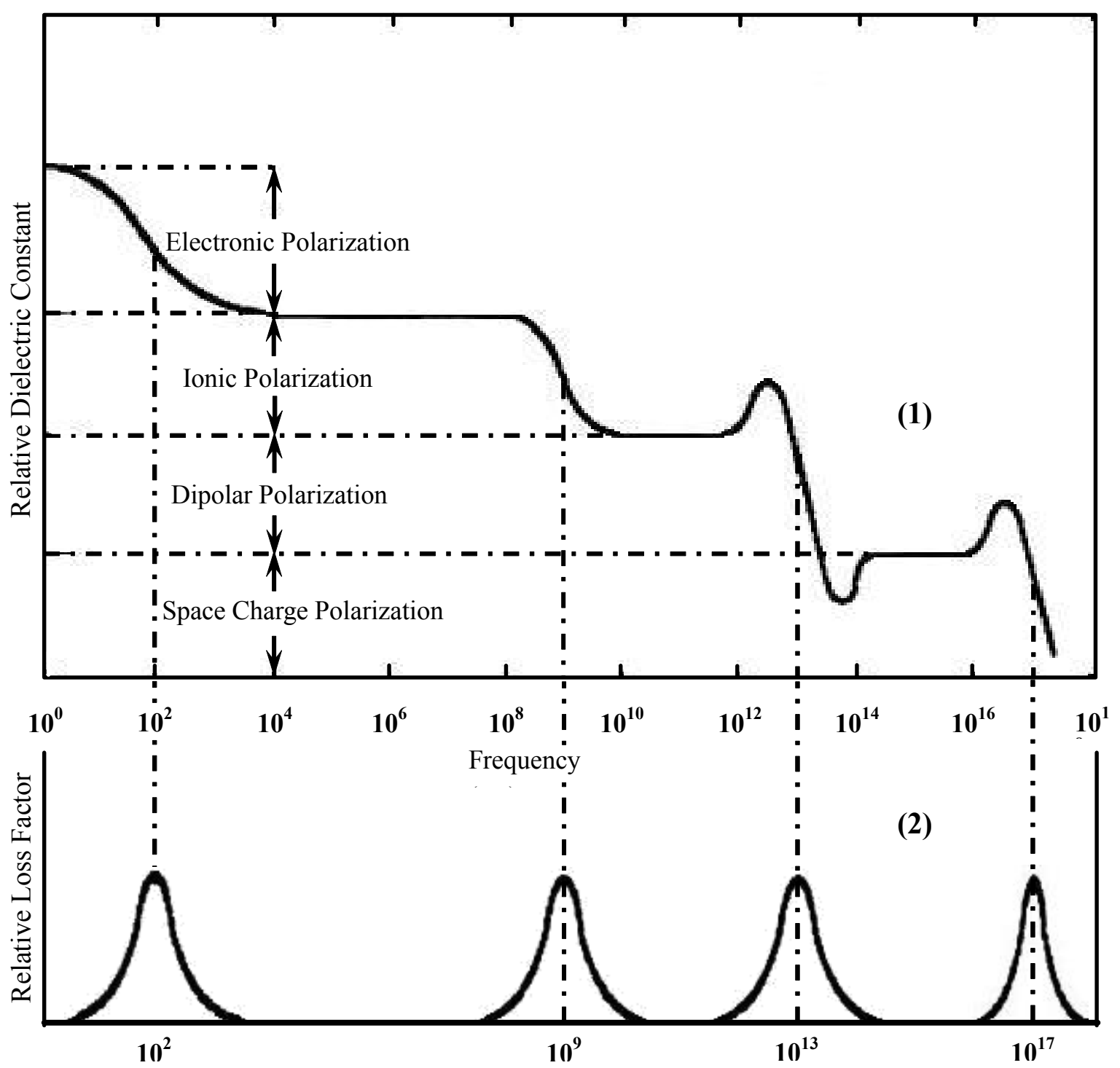

Figure 1. Dependence of 1) the relative dielectric constant and 2) the relative loss factor on frequency of an alternating electric Field. Contributions of electronic, ionic, dipolar and space charge in the total polarization are determined (source of this diagram: Ref. [25]).

temperature $77 \mathrm{~K}$ is much less than its maximum value attained at the working temperature $300 \mathrm{~K}$. Therefore, the RPDEC of the $\mathrm{Tl}_{2} \mathrm{~S}$ crystalline medium is strongly dependent on the temperature at the low frequency region.

2) Behavior in the second interval is observed in the high frequency region $28.3-68.6 \mathrm{KHz}$ in which the RPDEC of the $\mathrm{Tl}_{2} \mathrm{~S}$ crystalline medium starts falling slightly with the increase in frequency of the applied ac field till the value of $46.5 \mathrm{kHz}$ after which it seems close to the saturation phase. However, the values of the RPDEC at $68.6 \mathrm{kHz}$ were found to be 1224.51 and 1593.79 corresponding to the working temperatures 77 and $300 \mathrm{~K}$ respec- tively. So, the RPDEC of the $\mathrm{Tl}_{2} \mathrm{~S}$ crystalline medium shows a little dependence on both frequency and temperature at the high frequency region.

The rapid rise in RPDEC of the $\mathrm{Tl}_{2} \mathrm{~S}$ layered single crystals with decreasing frequency at low frequencies may be associated with the effect of the electrodes. However, the low values of RPDEC at high frequencies are important for these materials in the construction of photonic and NLO devices [26]. Furthermore, the higher value of the dielectric constant at low frequency in the crystals under study may be attributed to voids, dislocations and other defects [27]. It is reported in Ref. [28] that the large values of RPDEC are due to the fact that ferroelectric grains are surrounded by ferrite grains [29] 


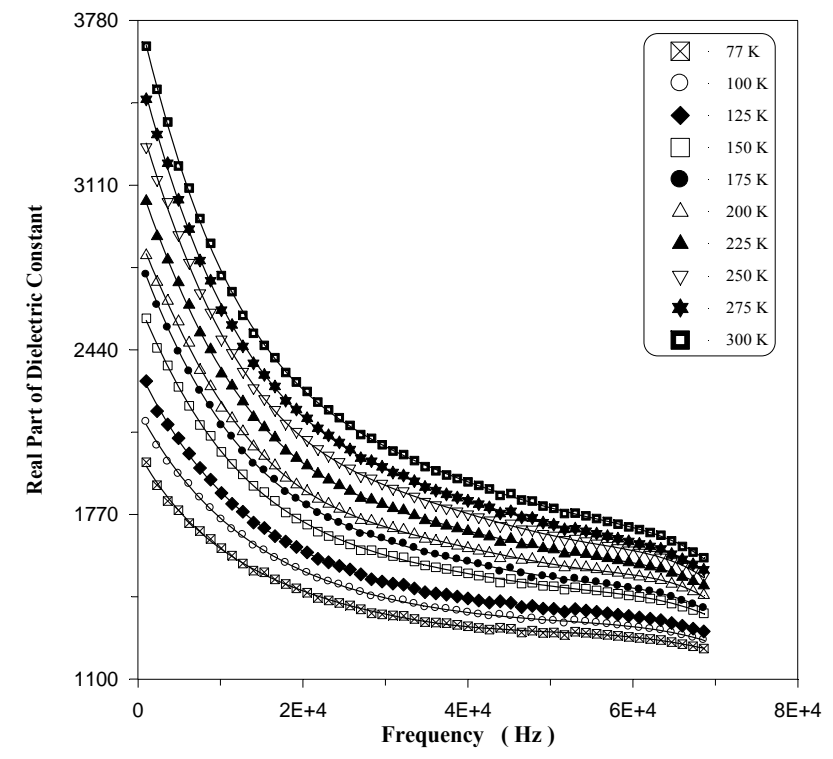

Figure 2. Shows the frequency dependence of the RPDEC for $\mathrm{Tl}_{2} \mathrm{~S}$ layered single crystals.

which in itself gives interfacial polarization [22]. The temperature dependence of the RPDEC for the $\mathrm{Tl}_{2} \mathrm{~S}$ crystalline medium was measured in the temperature range $77-300 \mathrm{~K}$ at different working frequencies of the applied ac field extending from 1 to $68.6 \mathrm{kHz}$. Behavior of the RPDEC as a function of temperature for the $\mathrm{Tl}_{2} \mathrm{~S}$ crystalline medium is shown in Figure 3. However, this figure involves the behavior for selected working frequencies ranging from 1 to $23.1 \mathrm{kHz}$ due to the similar effect of temperature on the RPDEC at all working frequencies investigated here. Based on this figure, a no

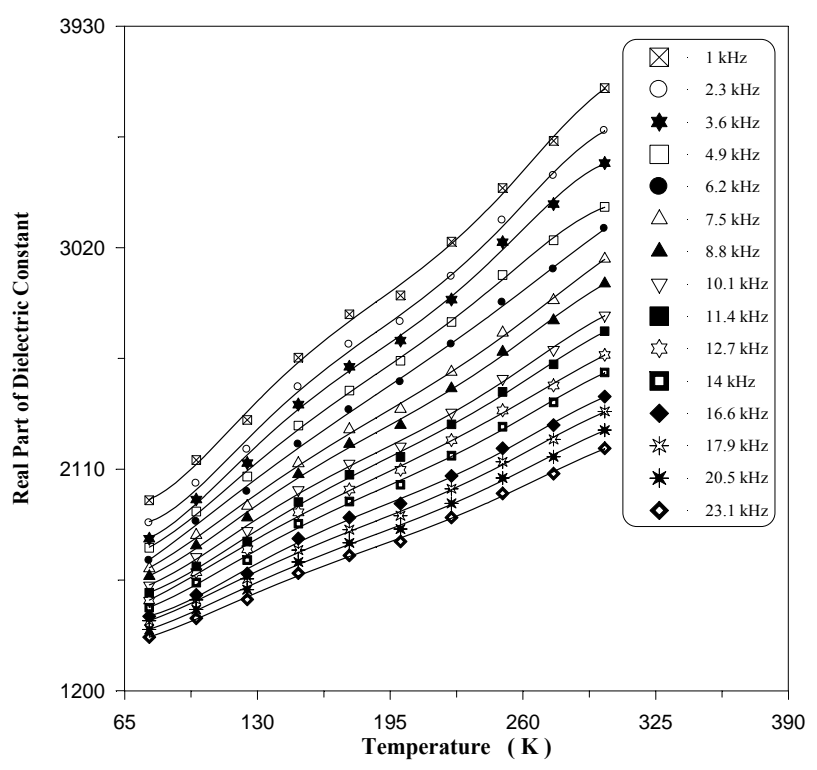

Figure 3. Temperature dependence of the RPDEC for $\mathrm{Tl}_{2} \mathrm{~S}$ layered single crystals. ticeable rise of the RPDEC commences from $77 \mathrm{~K}$ till the temperature of $300 \mathrm{~K}$ at all the investigated working frequencies. However, the maximum value of the RPDEC is obviously found to be at $300 \mathrm{~K}$ for all the investigated working frequencies. The maximum value was obviously found to be decreased from 3675.57 to 2195.57 corresponding to the working frequencies extending from 1 to $23.1 \mathrm{kHz}$. It is evident that the maximum value of the RPDEC at the working frequency 23.1 $\mathrm{kHz}$ is much less than its maximum value attained at the working frequency $1 \mathrm{kHz}$. Consequently, the RPDEC of the $\mathrm{Tl}_{2} \mathrm{~S}$ layered single crystals is strongly dependent on the frequency of the applied ac field at the high temperature region. However, Figure 3 shows an intensive rise of the RPDEC with temperature at all the investigated working frequencies leading to that the RPDEC of the $\mathrm{Tl}_{2} \mathrm{~S}$ layered single crystals is strongly dependent on temperature. Based on this figure, the RPDEC of the $\mathrm{Tl}_{2} \mathrm{~S}$ layered single crystals exhibits anomalous behavior with temperature at the low working frequencies that suggests the possibility of the crystal medium to be ferroelectric [22]. As the temperature rises, the interfacial polarization increases due to creation of crystal defects [30], where the increase in dielectric constant with increase in temperature is thus expected. In the current investigation, the sharp rise of RPDEC for the $\mathrm{Tl}_{2} \mathrm{~S}$ layered single crystals with temperature at all frequencies investigated here may be attributed to space charge (diffusional or interfacial) polarization [22]. Based on the frequency dependence of the RPDEC, the increase in capacitance and hence the dielectric constant towards the low frequency region has been attributed to an interfacial polarization mechanism [25,31]. An intensive in the RPDEC is clear in the low frequencies and could be due to the presence of the large capacitance at the electrode electrolyte interface, which apparently reduces the ac current [32].

\subsection{Influences of Frequency and Temperature on $\varepsilon^{\prime \prime}$}

The frequency dependence of the IPDEC for the $\mathrm{Tl}_{2} \mathrm{~S}$ layered single crystals was obtained in the frequency of the applied ac field ranging from 1-68.6 kHz at different working temperatures extending from 77 to $300 \mathrm{~K}$. The variation of the IPDEC with frequency of the applied ac field is illustrated in Figure 4. This figure shows a noticeable decrease in the IPDEC with frequency commences from $1 \mathrm{kHz}$ till the frequency of $68.6 \mathrm{kHz}$ at all the working temperatures investigated here. It is evident from Figure 4 that the behavior of the $\mathrm{Tl}_{2} \mathrm{~S}$ crystalline medium with respect to the IPDEC is similar to its behavior for RPDEC. However, behavior of the IPDEC for 


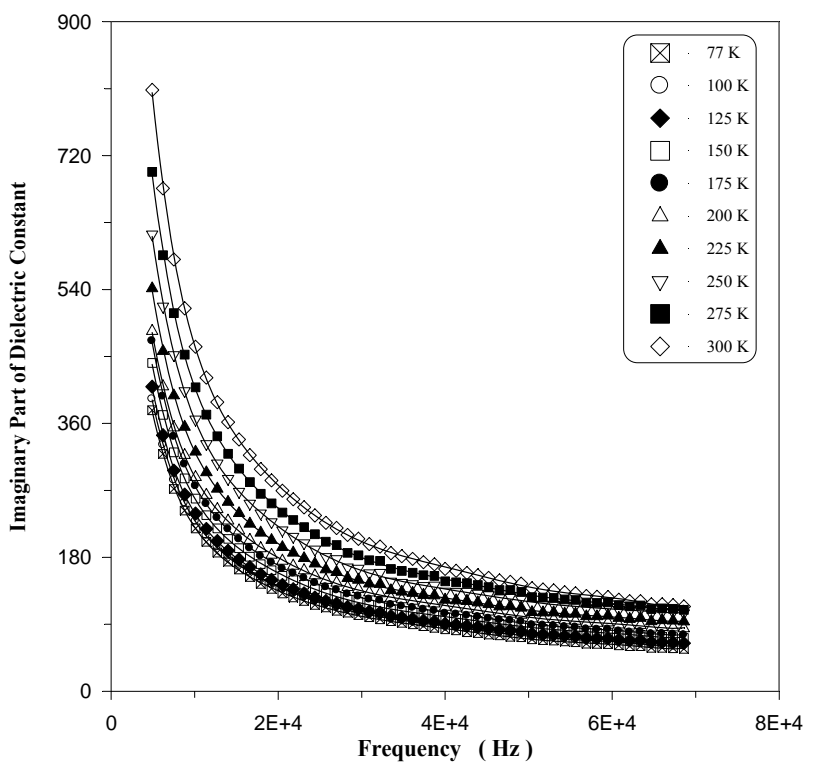

Figure 4. Shows the frequency dependence of the IPDEC for $\mathrm{Tl}_{2} \mathrm{~S}$ layered single crystals.

$\mathrm{Tl}_{2} \mathrm{~S}$ crystalline medium shows the greater dependencies on both frequency and temperature, and could be interpreted according to the frequency intervals:

1) First could be observed in the low frequency region 1-20.5 kHz: Behavior in this region shows a sharp decrease in the IPDEC with the increase in frequency. Hence, the IPDEC of the $\mathrm{Tl}_{2} \mathrm{~S}$ crystalline medium exhibits a strong dependence on the frequency at the low frequency region. The maximum value of the IPDEC is found to be at the beginning frequency for such a working temperature. Based on Figure 4, maximum value is found to be increased from 1430.59 to 3156.39 corresponding to the working temperatures ranging from 77 to $300 \mathrm{~K}$. Therefore, the maximum value of the IPDEC at the working temperature $77 \mathrm{~K}$ is much less than its maximum value attained at the working temperature $300 \mathrm{~K}$. So, the IPDEC of the $\mathrm{Tl}_{2} \mathrm{~S}$ crystalline medium is strongly dependent on the temperature at low frequency region.

2) The second could be observed in the high frequency region 20.5-68.6 KHz: Behavior in this region exhibits slightly falling in the IPDEC of the $\mathrm{Tl}_{2} \mathrm{~S}$ crystalline medium with the increase in frequency of the applied ac field starting from the frequency of 20.5 $\mathrm{kHz}$ till the value of $55.6 \mathrm{kHz}$. Thereafter, the value of the IPDEC seems close to the saturation phase for all working temperatures. So, the IPDEC of the $\mathrm{Tl}_{2} \mathrm{~S}$ crystalline medium shows a little dependence on frequency at the high frequency region. Furthermore, the values of the IPDEC at the frequency of $68.6 \mathrm{kHz}$ were found to be 56.87 and 113.81 corresponding to the working temperatures 77 and $300 \mathrm{~K}$ respectively. Hence, the IPDEC of the $\mathrm{Tl}_{2} \mathrm{~S}$ crystalline medium shows a weak dependence on temperature at the high frequency region compared with the dependence observed at the low frequency region.

The temperature dependence of the IPDEC for the $\mathrm{Tl}_{2} \mathrm{~S}$ layered single crystals was investigated in the temperature range $77-300 \mathrm{~K}$ at different working frequencies of the applied ac field extending from 1 to $68.6 \mathrm{kHz}$. The variation of the IPDEC with temperature is depicted in Figure 5. However, this figure involves the behavior for selected working frequencies ranging from 16.6 to 40 $\mathrm{kHz}$ due to the similar effect of temperature on the IPDEC at all working frequencies investigated here. This figure shows a considerable rise of the IPDEC commences from $77 \mathrm{~K}$ till the temperature of $300 \mathrm{~K}$ at the working frequencies ranging from 16.6 to $40 \mathrm{kHz}$. Figure 5 shows that in the low temperature region, the IP$\mathrm{DEC}$ of the $\mathrm{Tl}_{2} \mathrm{~S}$ crystalline medium increases slightly with the increase in temperature exhibiting a little dependence of the IPDEC on temperature in the low temperature region. However, the value of the IPDEC at the beginnings of the low temperature interval was found to be 153.53 and 83.18 corresponding to the working frequencies 16.6 and $40 \mathrm{kHz}$ respectively. So, the IPDEC of the $\mathrm{Tl}_{2} \mathrm{~S}$ crystalline medium shows a weak dependence on frequency in the low temperature region. Furthermore, the variation of the IPDEC with temperature in the high temperature region shows greater dependence on temperature than it variation in the low temperature region exhibiting a considerable dependence of the IPDEC on

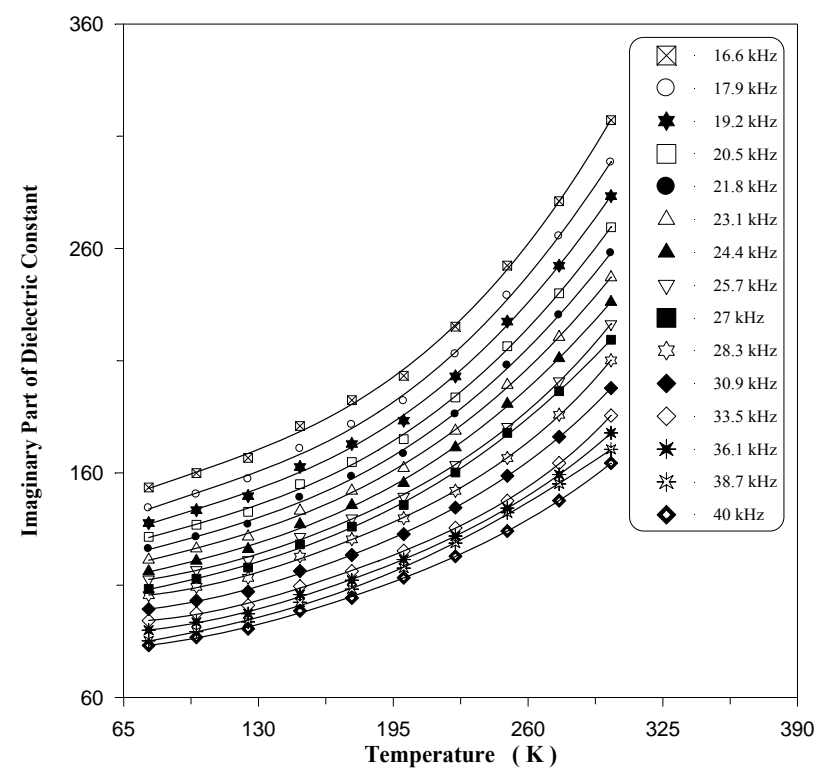

Figure 5. Shows the temperature dependence of the IPDEC for $\mathrm{Tl}_{2} \mathrm{~S}$ layered single crystals. 
temperature in the high temperature region. It is observed in the high temperature interval that the maximum value of the IPDEC is found to be at $300 \mathrm{~K}$ for all working frequencies investigated here. These maximum values of the IPDEC were found to be extended from 317.1 to 164.37 corresponding to the working frequencies ranging from 16.6 to $40 \mathrm{kHz}$. The maximum value of the IPDEC at the working frequency $40 \mathrm{kHz}$ seems much less than its maximum value attained at the working frequency 16.6 $\mathrm{kHz}$. Therefore, the IPDEC of the $\mathrm{Tl}_{2} \mathrm{~S}$ layered single crystals is strongly dependent on the temperature at the high temperature region. The large values of the dielectric constant and IPDEC exhibited by the $\mathrm{Tl}_{2} \mathrm{~S}$ layered single crystals at low frequencies may be ascribed to space charge polarization due to the crystalline defects [33]. Furthermore, the dielectric losses increase faster with the temperature at the low working frequencies that is clearly exhibited in Figure 3 at the low frequency region and could be due to the strongly defective structure and to the existence of shallow traps in the band gap [34]. The large value of the dielectric constant and IPDEC at the low frequency region (see Figures 3 and 4) may arises due to the presence of space charge polarization near the grain boundary interfaces which depends on the purity and perfection of the sample $[35,36]$. The contribution from space charge towards polarization depends on purity and perfection of crystals as the impurities and/or defects create potential barriers that limit the transport of charge carriers. So, the space charge (or diffusing) contribution involves a limited transport of charge carriers until they are stopped at a potential barrier, possibly a grain boundary or phase boundary [22]. However, space charge and dipolar polarizations are relaxation processes and are strongly temperature dependent [37].

\subsection{Influences of Frequency and Temperature on the Dissipation Factor}

The variation of the dissipation factor with frequency for the $\mathrm{Tl}_{2} \mathrm{~S}$ crystalline medium was measured in the frequency range 1-68.6 kHz at different working temperatures extending from 77 to $300 \mathrm{~K}$. Figure 6 shows the dissipation factor as a function of frequency exhibiting that the dissipation factor shows a considerable decreases with the increase in frequency form the beginning frequency till the frequency of $17.9 \mathrm{kHz}$. In this region of frequency, the dissipation factor exhibits strong dependence on frequency after which it decreases slightly with increasing frequency till the value of $54.3 \mathrm{kHz}$ at which the dissipation factor seems to be close to the saturation phase. So, the dissipation factor shows a little dependence on frequency at the high frequency region. Based on Figure 6, values of the dissipation factor at the begin-

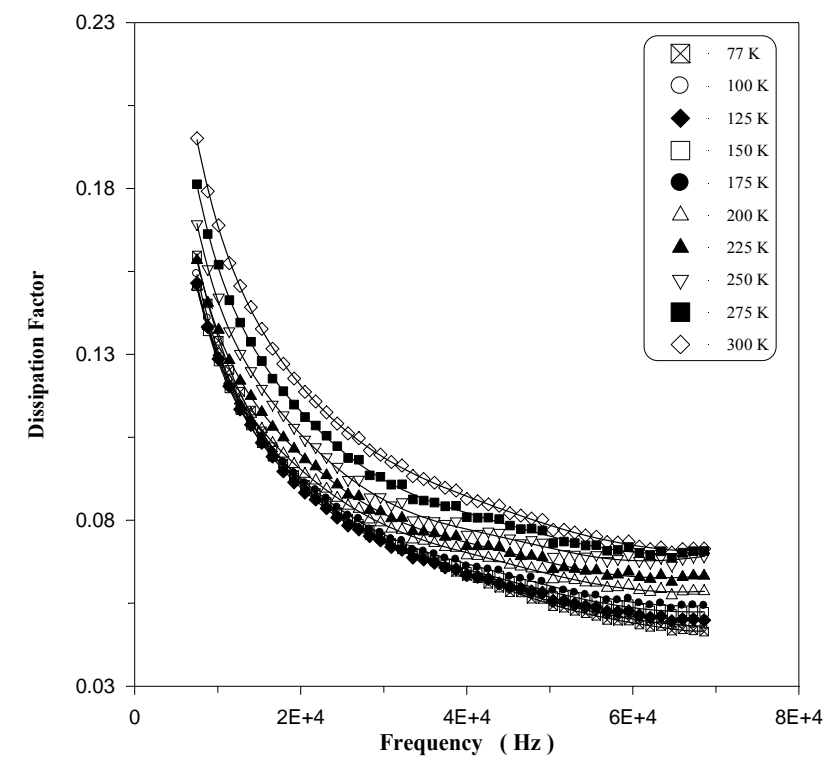

Figure 6. Frequency dependence of the dissipation factor for $\mathrm{Tl}_{2} \mathrm{~S}$ layered single crystals.

ning and ending frequencies for the working temperature of $77 \mathrm{~K}$ were calculated to be 0.72 and 0.046 respectively. However, these values of the dissipation factor were calculated for the working temperature of $300 \mathrm{~K}$ to be 0.85 and 0.071 respectively. The difference between the beginning values at the working temperature $77 \mathrm{~K}$ and that at the working temperature $300 \mathrm{~K}$ shows a weak dependence of the dissipation factor on temperature in the low frequency region. However, the same difference with respect to the ending values shows that the dissipation factor exhibits a greater dependence on temperature in the high frequency region than that is observed in the low frequency region.

The variation of the dissipation factor with temperature for the $\mathrm{Tl}_{2} \mathrm{~S}$ crystalline medium was measured in the temperature range $77-300 \mathrm{~K}$ at different working frequencies of the applied ac field extending from 1 to 68.6 kHz. However, Figure 7 shows the dissipation factor as a function of temperature with respect to selected working frequencies ranging from 16.6 to $33.5 \mathrm{kHz}$ due to the similar effect of temperature on the IPDEC at all working frequencies investigated here. Based on this figure, the dissipation factor shows a similar rise with increasing temperature at all the investigated working frequencies. In accordance with Figure 7, the dissipation factor exhibits a weak dependence on temperature in the low temperature region; however it shows an intensive increase with temperature in the high temperature region. Furthermore, this figure exhibits that there is a noticeable dependence of the dissipation factor on working temperatures, which coincides with the data described in Figure 6. Based on Figures 5-8, the IPDEC and dissipa- 


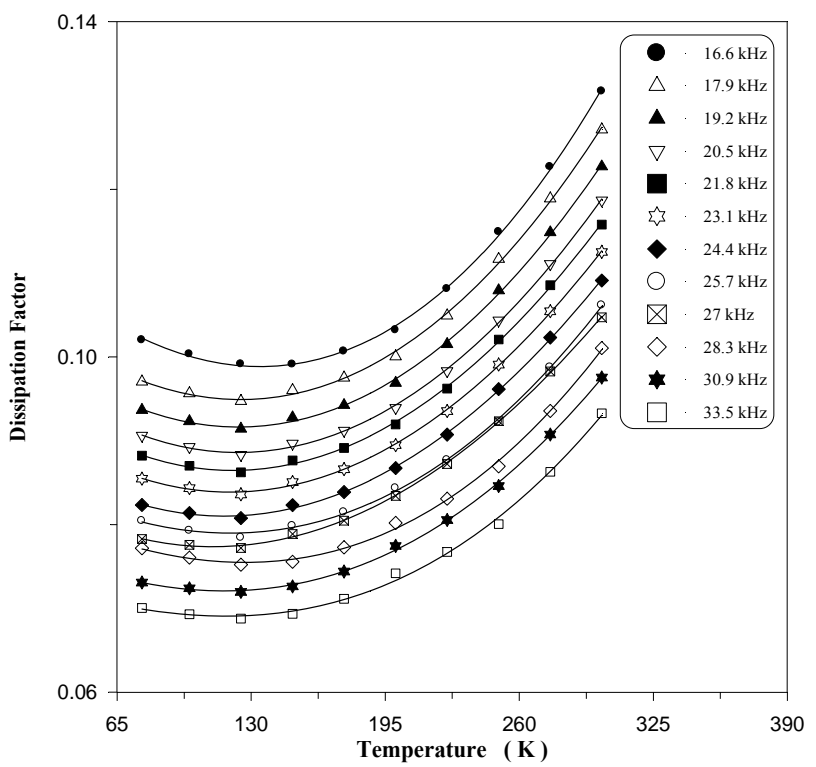

Figure 7. Temperature dependence of the dissipation factor for $\mathrm{Tl}_{2} \mathrm{~S}$ layered single crystals.

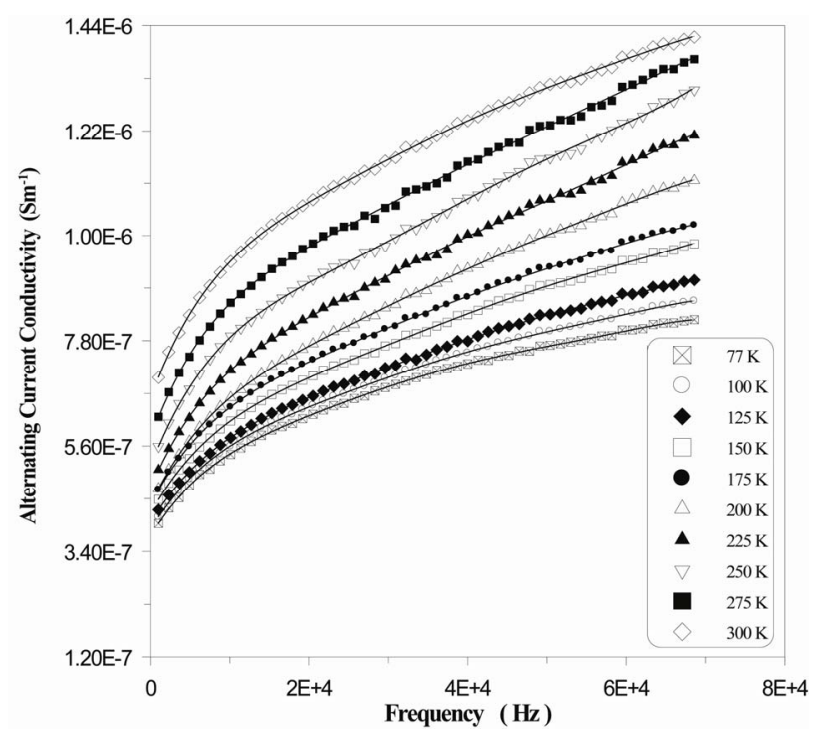

Figure 8. Shows the frequency dependence of the ac-C for the $\mathrm{Tl}_{2} \mathrm{~S}$ layered single crystals.

tion factor of the $\mathrm{Tl}_{2} \mathrm{~S}$ crystalline medium exhibit similar dependences on temperature and frequency of the applied ac electric field. Similarity in behavior has been observed for many materials like lanthanum heptamolybdate [38], neodymium heptamolybdate [39] and La-Nd heptamolybdate [40]. The increase in trend in $\tan \delta$ with temperature may be due to space charge polarization. Furthermore, the dielectric constant, dielectric loss, dissipation factor and conductivity are shown for the $\mathrm{Tl}_{2} \mathrm{~S}$ layered single crystals to be strongly dependent on temperature and frequency of the applied field. This has been applicable for a large number of materials [22]. The functional dependence of these dielectric parameters on temperature and frequency forms an important characteristic of the material and is required to be investigated. The dissipation factor of the $\mathrm{Tl}_{2} \mathrm{~S}$ crystalline medium material exhibits low values suggesting that the grown crystals possess good optical quality. However, this DE parameter is of vital importance for nonlinear optical materials in their applications [41].

\subsection{Influences of Frequency and Temperature on the ac-Conductivity}

Figure 8 illustrates the frequency dependence of the ac-C for the $\mathrm{Tl}_{2} \mathrm{~S}$ layered single crystals in the frequency range $1-68.6 \mathrm{kHz}$ at the working temperatures extending from 77 to $300 \mathrm{~K}$. For all the working temperatures investigated here, this figure shows a typical dependence of the ac-C for the $\mathrm{Tl}_{2} \mathrm{~S}$ layered single crystals on frequency. In accordance with Figure 8, the ac- $\mathrm{C}$ shows a noticeable increase with frequency in the low frequency region dependence exhibiting intensive dependence on frequency; however it shows weaker dependence on frequency in the high temperature region than that is observed in the low frequency region. In accordance with Figure 8, values of the ac-C at the beginning and ending frequencies for the working temperature of $77 \mathrm{~K}$ were calculated to be $3.98 \times 10^{-7}$ and $8.23 \times 10^{-7}$ respectively. However, these values of the ac-C were calculated for the working temperature of $300 \mathrm{~K}$ to be $7.03 \times 10^{-7}$ and $1.42 \times 10^{-6}$ respectively. The difference between the beginning values at the working temperatures 77 and $300 \mathrm{~K}$ shows a weak dependence of the ac-C on temperature in the low frequency region. However, the same difference with respect to the ending values shows that the ac-C exhibits a strong dependence on temperature in the high frequency region compared with that is observed in the low frequency region. It is also clear from Figure 8 that the behavior of the ac-C for the $\mathrm{Tl}_{2} \mathrm{~S}$ layered single crystals, as a function of frequency, exhibits three zones. The first is observed in the low frequency region in which the ac- $\mathrm{C}$ increases linearly with increasing frequency; the second zone shows a non-linear region that is followed by the third zone in which the ac-C increases linearly with the increase in frequency with smaller slope that is observed in the low frequency region. The linear zones were observed for all the working temperatures investigated here that coincides with what is reported in Ref. [42] that the ac-C increases linearly with increasing frequency for most semiconductors. In accordance with this Figure 8, the dependence of the measured ac-C with frequency of the applied ac field at all working temperatures investigated here could be formulated as [26]: 


$$
\sigma(\omega)=\sigma(0)+A \omega^{S}
$$

where $\sigma(0)$ is the dc conductivity, A is the pre-exponenttial factor and $\mathrm{s}$ is the exponent. The aforementioned equation is referred as the universal dynamic pattern of ac electrical behavior of conducting solids and liquids as proposed by Jonscher [43] based on the exponent $\mathrm{s}$ lies in the range $0<\mathrm{s}<1$. It has been used mostly to characterize the electrical conduction in disorder ionic glasses, amorphous semiconductors, ionic conductors and some crystals [44-50]. This power law is related to the dynamics of hopping transport between states in the forbidden gap. The exponent $\mathrm{s}$ is the measure of the degree of interaction with the environment. Experimental evidence of this behavior is a power law of the ac conductivity $\sigma_{a c}(\omega)=A \omega^{s}$ observed over many decades of frequency [51]. The interpretation usually involves analysis of the temperature dependence of $s(T)$ which makes it possible to find the relevance of the hopping mechanism in terms of pair approximation [47]. Based on the aforementioned power law, the quantity $\ln \sigma_{a c}$ was plotted as a function of $\ln \omega$ that is depicted in Figure 9. In line with this figure, the values of the exponent $\mathrm{s}$ for all working temperatures were evaluated by calculating the slopes of resultant straight lines. In the current work, the estimated values of the exponent $s$ were found to be less than unity in the range $0.21 \geq \mathrm{s} \geq$ 0.19 . The values of the exponent $s$ were plotted versus temperature for all frequency investigated here to describe the temperature impact on it and determining its temperature coefficient (see Figure 10). The figure shows that in the low temperature region ( 77 to $175 \mathrm{~K}$ ), the exponent $\mathrm{s}$ is found to be frequency dependent and it increases line-

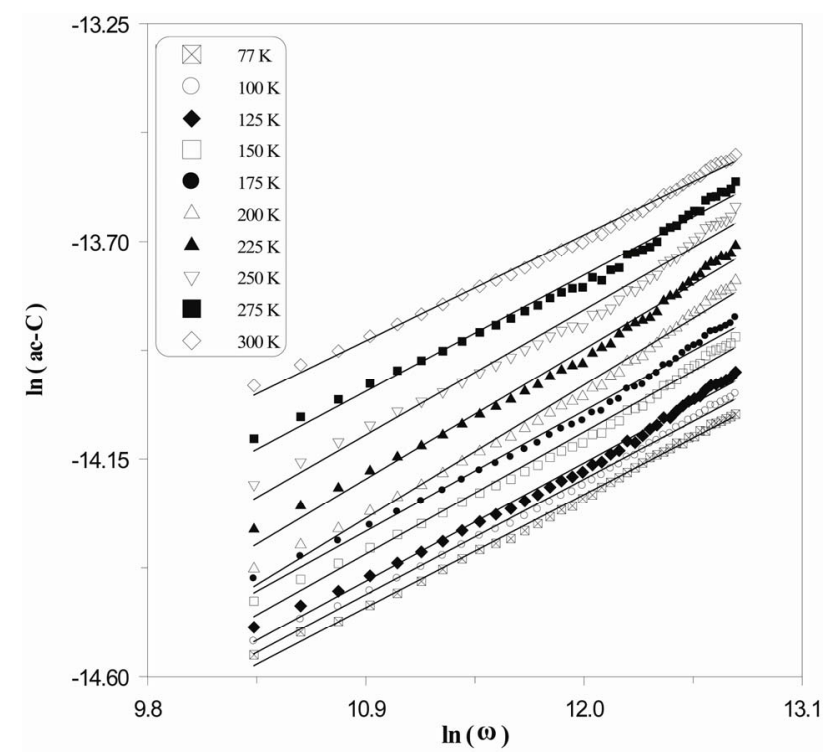

Figure 9. Plot of the quantity $\ln \left(\sigma_{a c}\right)$ as a function of $\ln (\omega)$ for $\mathrm{Tl}_{2} \mathrm{~S}$ layered single crystals.

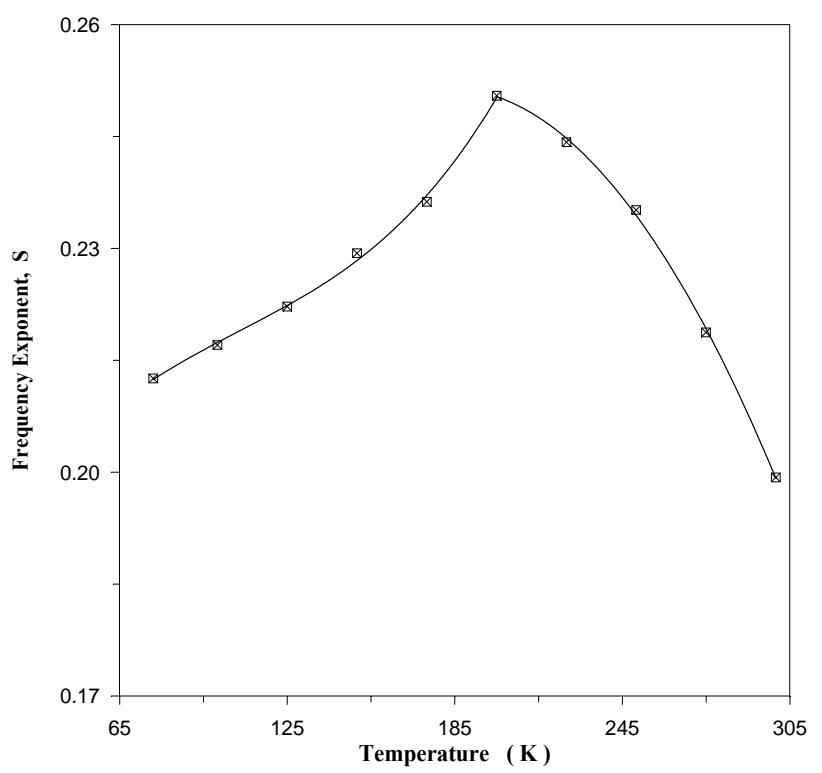

Figure 10. The temperature dependence of the exponent $s$ for $\mathrm{Tl}_{2} \mathrm{~S}$ layered single crystals.

arly as the working temperature increases with a temperature coefficient of about $2.94 \times 10^{-4}$. However in the high temperature region 175 to $300 \mathrm{~K}$, the exponent s decreases linearly as the working temperature increases with a temperature coefficient of about $-5.11 \times 10^{-4}$.

The ac-C of the $\mathrm{Tl}_{2} \mathrm{~S}$ layered single crystals was evaluated by substituting the values of $\varepsilon$ and $\tan \delta$ in Equation 2. The temperature dependence of the ac- $\mathrm{C}$ was investigated in the temperature range $77-300 \mathrm{~K}$ at the working frequencies ranging from 1 to $68.6 \mathrm{kHz}$. However, Figure 11 shows the ac-C as a function of temperature

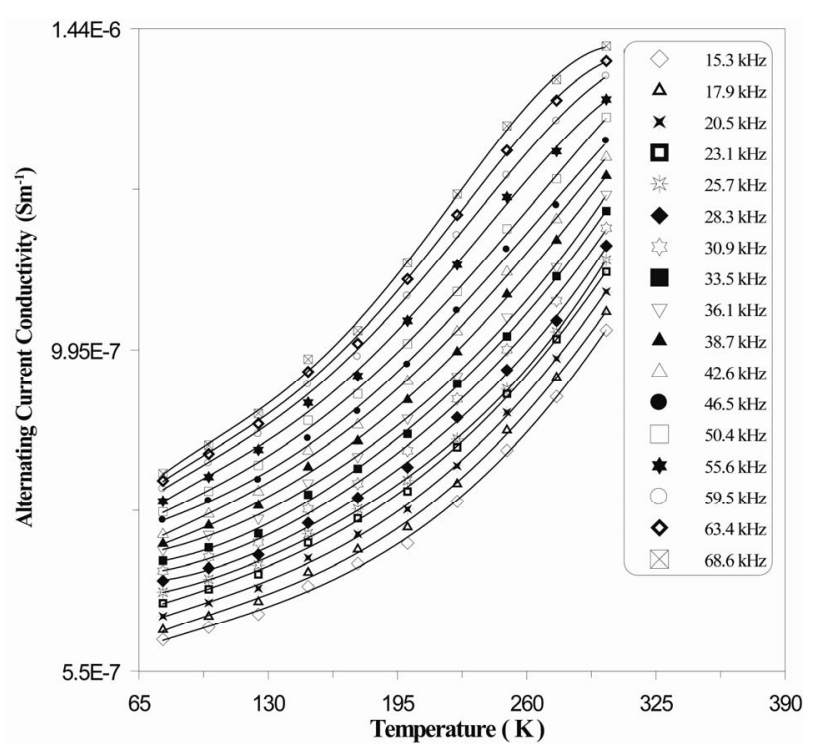

Figure 11. Shows the temperature dependence of the ac-C for $\mathrm{Tl}_{2} \mathrm{~S}$ layered single crystals. 
with respect to selected working frequencies ranging from 15.3 to $68.6 \mathrm{kHz}$. Based on this figure, a typical dependence of the ac-C on temperature is observed at all working frequencies investigated here. In addition, the figure shows that the ac- $\mathrm{C}$ exhibit a noticeable increase with increasing temperature in the low temperature region at all investigated working frequencies. Whereas, the ac-C fulfills an intensive rise with increasing temperature in high temperature region at all investigated working frequencies. The later leads to that the ac-C of the $\mathrm{Tl}_{2} \mathrm{~S}$ layered single crystals shows strong dependence on temperature at all investigated working frequencies.

It is obvious from Figures $(3-8,9,12)$ that $T$ he $\mathrm{Tl}_{2} \mathrm{~S}$ layered single crystals exhibit an intensive dependence of RPDEC, IPDEC, dissipation factor and ac-C on temperature and frequency of the applied ac field (see Table 1). So, the functional dependence of these DE parameters on temperature and frequency forms an important characteristic of these materials and is strongly required to be investigated. However, strong dependencies of these DE parameters on temperature and frequency of the applied ac field have been applicable for a large number of materials [22, 38-40].

Figure 12 depicts a plot of the quantity $\ln \sigma$ as a function of $1000 / \mathrm{T}$ in the temperature range $77-300 \mathrm{~K}$ at selected working frequencies extending from 1 to 20.5 $\mathrm{kHz}$. This graph was plotted based on an Arrhenius plot using the following relation [52]:

$$
\sigma=\sigma_{0} \exp \left(-\frac{E_{a}}{k T}\right)
$$

where $\sigma$ is the conductivity at temperature $T, \mathrm{E}_{\mathrm{a}}$ the activation energy for the ac electrical process, and $\mathrm{k}$ the Boltzmann's constant. In accordance wit the reference investigation [34], Figure 9 suggests that the condition

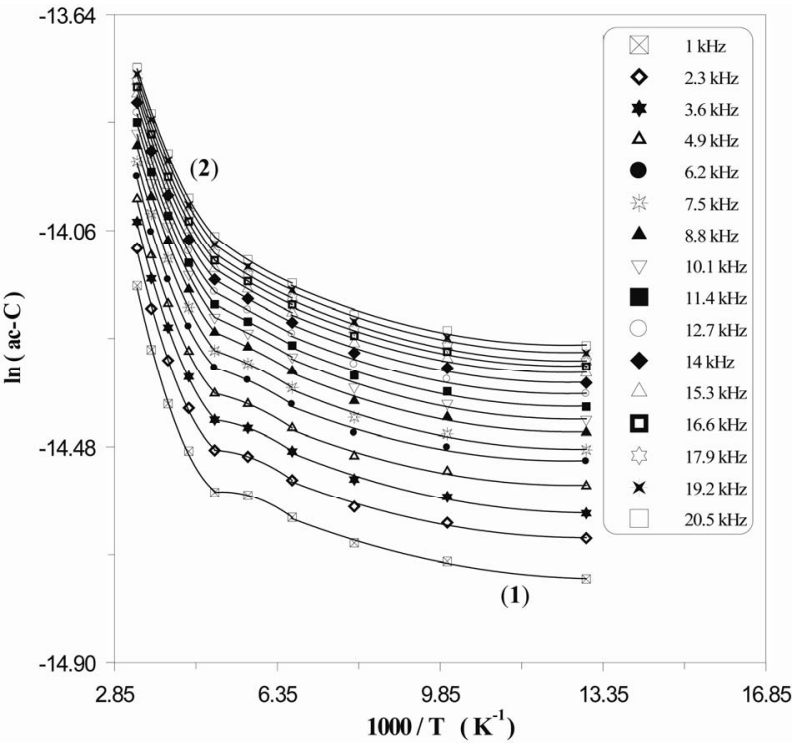

Figure 12. Shows the inversely dependence of the quantity In (ac-C) on temperature for the layered $\mathrm{Tl}_{2} \mathrm{~S}$ single crystals.

in line with the aforementioned power law (Equation 5) is satisfied for the $\mathrm{Tl}_{2} \mathrm{~S}$ layered single crystals, and hence the charge carriers are assumed to be transferred by hopping between localized states and shallow traps in the band gap. So the charge transfer process can be described by two mechanisms: 1) Hopping near the periphery of the conduction band or valence zone that is characterized by a strong temperature dependence of exponential behavior; and 2) Hopping between localized states with energies close to the Fermi-Level in which a weak temperature dependence is observed.

Furthermore, Figure 12 shows that in the temperature region I (77 to $200 \mathrm{~K}$ ), the charge transfer process could

Table 1. A resultant data showing the variation of RPDEC, IPDEC and the dissipation factor with frequency and temperature.

\begin{tabular}{|c|c|c|c|c|c|c|}
\hline \multirow{2}{*}{$\begin{array}{l}\text { Measuring Frequency } \\
(\mathbf{k H z})\end{array}$} & \multicolumn{2}{|c|}{ Real Part of Dielectric Constant } & \multicolumn{2}{|c|}{$\begin{array}{c}\text { Imaginary Part of Dielectric Con- } \\
\text { stant }\end{array}$} & \multicolumn{2}{|c|}{ Dissipation Factor } \\
\hline & At $T=77 \mathrm{~K}$ & At $T=300 \mathrm{~K}$ & At $T=77 \mathrm{~K}$ & At $T=300 \mathrm{~K}$ & At $T=77 \mathrm{~K}$ & At $T=300 \mathrm{~K}$ \\
\hline 1 & 1982.586 & 3675.57 & 1430.593 & 3156.392 & 0.72158 & 0.858749 \\
\hline 6.2 & 1734.293 & 3098.165 & 318.9989 & 675.8933 & 0.183936 & 0.218159 \\
\hline 11.4 & 1602.343 & 2676.65 & 200.785 & 421.4868 & 0.125307 & 0.157468 \\
\hline 16.6 & 1505.173 & 2408.117 & 153.5354 & 317.1067 & 0.102005 & 0.131682 \\
\hline 21.8 & 1429.978 & 2227.362 & 126.1257 & 257.9126 & 0.088201 & 0.115793 \\
\hline 27 & 1383.71 & 2094.569 & 108.2979 & 219.3343 & 0.078266 & 0.104716 \\
\hline 32.2 & 1357.331 & 2013.871 & 96.71062 & 194.3052 & 0.071251 & 0.096483 \\
\hline 37.4 & 1327.718 & 1930.198 & 87.43122 & 173.2954 & 0.065851 & 0.089781 \\
\hline 42.6 & 1301.227 & 1873.673 & 79.20938 & 158.8382 & 0.060873 & 0.084774 \\
\hline 47.8 & 1296.91 & 1824.514 & 73.06217 & 146.8826 & 0.056336 & 0.080505 \\
\hline 53 & 1292.594 & 1775.356 & 68.14545 & 135.4191 & 0.05272 & 0.076277 \\
\hline 58.2 & 1275.748 & 1730.433 & 63.45999 & 126.7571 & 0.049743 & 0.073252 \\
\hline 63.4 & 1258.901 & 1685.509 & 60.55789 & 120.9542 & 0.048104 & 0.071761 \\
\hline 68.6 & 1224.51 & 1593.799 & 56.87902 & 113.816 & 0.04645 & 0.071412 \\
\hline
\end{tabular}


be due to hopping conduction between localized states with energies close to the Fermi-Level. However in the temperature region II ( 200 to $300 \mathrm{~K}$ ), the ac-C exhibits a strong increase with increasing temperature that suggests the prevailing of hopping process between localized states near the valence or conduction zone. In the temperature region II (200 to $300 \mathrm{~K})$, the measured ac-C is temperature dependent and less frequency dependent. The slope of this region is higher than the previous region. The influence of temperature on ac conductivity may be explained by considering the mobility of charge carriers responsible for hopping. Whereas, temperature increases the mobility of hopping carriers that causes thereby increase in conductivity $[31,53]$. In accordance with Figure 12, the inversely dependence of the quantity $\ln \sigma$ exhibits two straight lines with different slopes at such a working frequency. So, the activation energy of the electrical processes for the $\mathrm{Tl}_{2} \mathrm{~S}$ layered single crystals could be evaluated with respect to the temperature intervals. In the low temperature region, the activation energy of the ac electrical processes for the $\mathrm{Tl}_{2} \mathrm{~S}$ layered single crystals was calculated with respect to the working frequencies investigated here to be found in the range $0.0203 \mathrm{eV} \geq$ $\Delta E_{\mathrm{ac}} \geq 0.0223 \mathrm{eV}$. In this region, it is remarked that the activation energy of the ac electrical processes for the $\mathrm{Tl}_{2} \mathrm{~S}$ layered single crystals depends randomly on temperature. However activation energy was estimated in the high temperature region to be found in the range $0.22 \mathrm{eV}$ $\geq \Delta E_{\mathrm{ac}} \geq 0.261 \mathrm{eV}$. This region shows a regular dependence for the activation energy of the ac electrical processes on temperature, where it is decreased with the increase in frequency (see Figure 13). The complete data

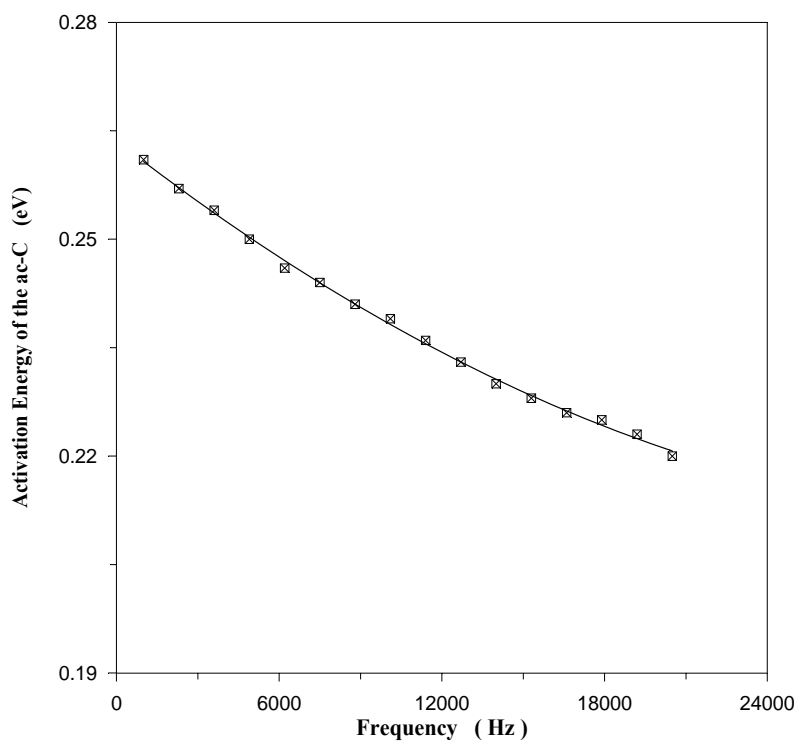

Figure 13. Shows the frequency dependence of the activation energy of the ac-C for the $\mathrm{Tl}_{2} \mathrm{~S}$ layered single crystals.

concerning the activation energy of the ac electrical processes for the $\mathrm{Tl}_{2} \mathrm{~S}$ layered single crystals is reported in Table 2. It is evident form Figure 13 that $\Delta \mathrm{E}_{\sigma}(\omega)$ tends to decrease with increasing frequency. Such a decrease in the activation energy with the frequency could be attributed to the increase of the applied ac - electric field that enhance the electronic jump between the localized states [24]. This behavior of the variation of $\Delta \mathrm{E}_{\sigma}(\omega)$ with frequency has been observed in several materials [54-56].

Table 2. A compiled data of the electrical processes in an applied ac electric field.

\begin{tabular}{|c|c|c|c|c|c|c|}
\hline & & \multirow{2}{*}{$\begin{array}{l}\text { Frequency } \\
\quad(\mathbf{k H z})\end{array}$} & \multicolumn{2}{|c|}{$\begin{array}{l}\text { The ac Activation Energy } \\
\qquad \Delta \mathbf{E}_{\mathrm{ac}}(\omega) \text { in } \mathrm{eV}\end{array}$} & \multicolumn{2}{|c|}{$\begin{array}{l}\text { The ac Conductivity } \\
\qquad \sigma_{\mathrm{ac}} \text { in } \mathrm{Sm}^{-1}\end{array}$} \\
\hline \multirow{5}{*}{\multicolumn{2}{|c|}{$\begin{array}{l}\text { Investigated } \\
\text { Frequency range } \\
1 \text { to } 68.6 \mathrm{kHz}\end{array}$}} & & $\begin{array}{c}\text { Phase I } \\
(200 \text { to } 300 \mathrm{~K})\end{array}$ & $\begin{array}{c}\text { Phase II } \\
\text { (77 to } 200 \mathrm{~K})\end{array}$ & $\begin{array}{c}\text { At } \\
T=77 \mathrm{~K}\end{array}$ & $\begin{array}{c}\text { At } \\
\mathbf{T}=\mathbf{3 0 0} \mathbf{K}\end{array}$ \\
\hline & & 1 & 0.261 & 0.0215 & $3.981 \mathrm{E}-07$ & $7.031 \mathrm{E}-07$ \\
\hline & & 2.3 & 0.257 & 0.0208 & $4.306 \mathrm{E}-07$ & $7.563 \mathrm{E}-07$ \\
\hline & & 4.9 & 0.25 & 0.0206 & $4.771 \mathrm{E}-07$ & $8.331 \mathrm{E}-07$ \\
\hline & & 6.2 & 0.246 & 0.0203 & $5.003 \mathrm{E}-07$ & $8.695 \mathrm{E}-07$ \\
\hline $\mathbf{T}(\mathrm{K})$ & $\mathbf{s}$ & 7.5 & 0.244 & 0.0218 & $5.115 \mathrm{E}-07$ & $8.948 \mathrm{E}-07$ \\
\hline 77 & 0.212 & 8.8 & 0.241 & 0.0217 & $5.288 \mathrm{E}-07$ & $9.225 \mathrm{E}-07$ \\
\hline 100 & 0.217 & 10.1 & 0.239 & 0.0221 & $5.421 \mathrm{E}-07$ & $9.454 \mathrm{E}-07$ \\
\hline 125 & 0.222 & 11.4 & 0.236 & 0.0217 & $5.557 \mathrm{E}-07$ & $9.654 \mathrm{E}-07$ \\
\hline 175 & 0.236 & 14 & 0.23 & 0.0215 & $5.82 \mathrm{E}-07$ & $1.005 \mathrm{E}-06$ \\
\hline 200 & 0.25 & 15.3 & 0.228 & 0.0214 & $5.935 \mathrm{E}-07$ & $1.022 \mathrm{E}-06$ \\
\hline 225 & 0.244 & 16.6 & 0.226 & 0.0218 & $6.008 \mathrm{E}-07$ & $1.036 \mathrm{E}-06$ \\
\hline 250 & 0.235 & 17.9 & 0.225 & 0.0223 & $6.068 \mathrm{E}-07$ & $1.048 \mathrm{E}-06$ \\
\hline 275 & 0.218 & 19.2 & 0.223 & 0.0222 & $6.169 \mathrm{E}-07$ & $1.063 \mathrm{E}-06$ \\
\hline 300 & 0.199 & 20.5 & 0.22 & 0.0219 & $6.26 \mathrm{E}-07$ & $1.075 \mathrm{E}-06$ \\
\hline
\end{tabular}




\section{Conclusion}

The RPDEC of the $\mathrm{Tl}_{2} \mathrm{~S}$ layered single crystals exhibits anomalous behavior with temperature at all the working frequencies investigated here that suggests the possibility of the crystal medium to be ferroelectric. Furthermore, sharp rise in the RPDEC with temperature at all investigated frequencies may be attributed to space charge (diffusional or interfacial) polarization. However, the increase in capacitance and hence the dielectric constant towards the low frequency region could be attributed to an interfacial polarization mechanism. The IPDEC of the $\mathrm{Tl}_{2} \mathrm{~S}$ layered single crystals exhibits strong dependence on temperature at the high temperature region and shows large values at low frequencies that may be ascribed to space - charge polarization due to the crystalline defects. The dielectric losses increase faster with temperature at the low working frequencies that could be due to the strongly defective structure and to the existence of shallow traps in the band gap. The experimental results of the ac-C measurements for the $\mathrm{Tl}_{2} \mathrm{~S}$ layered single crystals show that in the temperature region I ( 77 to $200 \mathrm{~K}$ ), the charge transfer process could be due to hopping conduction between localized states with energies close to the Fermi - Level. However in the temperature region II (200 to $300 \mathrm{~K}$ ), the ac-C exhibits a strong increase with increasing temperature that suggests the prevailing of hopping process between localized states near the valence or conduction zone. The influence of temperature on ac conductivity may be explained by considering the mobility of charge carriers responsible for hopping. Whereas, temperature increases the mobility of hopping carriers that causes thereby increase in conductivity. The dielectric constant, dielectric loss, dissipation factor and conductivity for the $\mathrm{Tl}_{2} \mathrm{~S}$ layered single crystals shows strong dependence on both the temperature and frequency of the applied field that has been applicable for a large number of materials. So, the functional dependence of these DE parameters on temperature and frequency forms important characteristics of such a material and is required to be investigated. The dissipation factor of the $\mathrm{Tl}_{2} \mathrm{~S}$ crystalline medium material exhibits low values suggesting that the grown crystals possess good optical quality. However, this DE parameter is of vital importance for nonlinear optical materials in their applications.

\section{References}

[1] S. Radtke and F. W. Dickson, "Carlinite, TI2S, a New Mineral from Nevada," American Mineralogist, Vol. 60, 1975, pp. 559-565.

[2] J. A. A. Ketelaar, E. W. Gorter and Z. Kristallogr, Vol. 101, 1939, p. 367.
[3] L. I. Man, Soviet Physics - Crystallography, Vol. 15, 1970, p. 399.

[4] G. Giester, C. L. Lengauer, E. Tillmanns and J. Zemann, " $\mathrm{Tl}_{2} \mathrm{~S}$ : Re-Determination of Crystal Structure and Stereochemical Discussion," Journal of Solid State Chemistry, Vol. 168, 2002, p. 322. doi:10.1006/jssc.2002.9711

[5] S. M. El-Sayed and S. A. Fayek, "Low Temperature Dielectric Behavior and AC Conductivity in Metal-containing Chalcogenide $\mathrm{Ge}_{2} \mathrm{~S}_{3}$ Films," Solid State Ionics, Vol. 176, 2005, pp. 149-154. doi:10.1016/j.ssi.2004.07.004

[6] R. A. Suleymanov, S. Ellialtioglu and B. G. Akinoglu, "Layered Semiconductor GeS as Birefringent Stratified Medium," Physical Review B, Vol. 52, 1995, 7806.

doi:10.1103/PhysRevB.52.7806

[7] R. Clasen, G. Harbeke, A. Korst, F. Levy, O. Madelung, K. Masclike, G. Nimtz, B. Schlict, F. J. Schlicht, F. J. Schmittle and J. Treusch, "Landolt-Bornstein, Numerical Data and Functional Relationships in Science and Technology," In: K. H. Hellwege and O. Madelung, Eds., Springer, Berlin, 1983, p. 94.

[8] G. Morgaritondo, "Electronic Structure and Electronic Transition in Layer Materials," In: V. Grasso, Ed., Physics and Chemistry of Materials with Low Dimensional Structure, Reidel, Dordrecht, 1986.

[9] G. D. Guseinov, E. Mooser, E. M. Kerimova, R. S. Gamidov, I. V. Alekseev and M. Z. Ismailov, "On Some Properties of TlInS $\mathrm{S}_{2}\left(\mathrm{Se}_{2}, \mathrm{Te}_{2}\right)$ Single Crystals," Physica Status Solidi, Vol. 34, 1969, p. 33. doi:10.1002/pssb.19690340103

[10] K. K. Mamedov, A. M. Adbullaev and E. M. Kerimova, "Heat Capacities of TlInS ${ }_{2}$ and TlInSe ${ }_{2}$ Crystals at Low Temperatures," Physica Status Solidi A, Vol. 94, 1986, p. 115. doi:10.1002/pssa.2210940112

[11] G. D. Guseinov, A. U. Malsagov, A. Kh. Matiev S. K. Umarov, E. G. Abdullaev and M. L. Shubnikov, Soviet Physics Semiconductors, Vol. 19, 1985, p. 445.

[12] N. M. Gasanly, N. N. Menlik, A. S. Ragimov and V. I. Tagirov, Soy. Phys. Solid State, Vol. 26, 1984, p. 336.

[13] K. R. Allakhverdiev, S. S. Babaev, N. A. Bakhyshov, T. G. Mamedov, E. Yu. Salaev and E. K. Efendieva, "The Influence of Hydrostatic Pressure on the Fundamental Absorption Edge of Crystals with T1Se-Type Structure," Physica Status Solidi, Vol. 126, 1984, K139. doi: $10.1002 /$ pssb.2221260244

[14] W. Henkel, H. D. Hochheimer, C. Carlone, A. Werner, S. Ves and H. G. V. Vonscg, "High-pressure Raman Study of the Ternary Chalcogenides $\mathrm{TlGaS}_{2}, \mathrm{TlGaSe}_{2}, \mathrm{TlInS}_{2}$, and TlInSe 2 ," Physical Review B, Vol. 26, 1982, p. 3211. doi:10.1103/PhysRevB.26.3211

[15] S. N. Mustafaeva, M. M. Asadov and K. S. Qahramanov, "Frequency-Dependent Dielectric Coefficients of T1InS ${ }_{2}$ Amorphous Films," Semiconductor Physics, Quantum Electronics \& Optoelectronics, Vol. 10, No. 2, 2007, pp. 58-61.

[16] S. N. Mustafaeva, M. M. Asadov and V. A. Ramazanzade, "Dielectric Properties and Ac-conductivity of TlInS ${ }_{2}$ Single Crystals," Fizika Tverdogo Tela, Vol. 38, No. 1, 1996, 
pp. 14-18.

[17] S. N. Mustafaeva, V. A. Aliev, M. M. Asadov, "DC Hopping Conduction in $\mathrm{TlGaS}_{2}$ and $\mathrm{TlInS}_{2}$ Single Crystals," Fiz. Tverd. Tela, Vol. 40, No. 4, 1998, pp. 612-615.

[18] E. Şentürk, "Dielectric Characteristics of a $\mathrm{Ce}^{3+}$-Doped $\mathrm{Sr}_{0.61} \mathrm{Ba}_{0.39} \mathrm{Nb}_{2} \mathrm{O}_{6}$ Single Crystal with Cole-Cole Plots Technique," Journal of Solid State Chemistry, Vol. 177, No. 4-5, 2004, pp. 1508-1512. doi:10.1016/j.jssc.2003.12.001

[19] H. A. Elshaikh, I. M. Ashraf and A. M. Badr, "Special Technique for Growing $\mathrm{Tl}_{4} \mathrm{~S}_{3}$, Anisotropy of Electrical Conduction and Photophysical Properties," Journal of Physical Chemistry B, Vol. 108, 2004, pp. 11327-11332. doi:10.1021/jp031035z

[20] I. Chaus et al., Russian Journal of Inorganic Chemistry, Vol. 24, 1979, p. 346.

[21] K. A. Narula, R. Singh and S. Chandra, "Low Frequency $\mathrm{AC}$ Conduction and Dielectric Relaxation in Poly(N-methyl Pyrrole)," Bulletin of Materials Science, Vol. 23, No. 3, 2000, pp. 227-232.

[22] B. Lal, S. K. Khosa, R. Tickoo, K. K. Bamzai and P. N. Kotru, "Dielectric Characteristics of Melt Grown Doped $\mathrm{KMgF}_{3}$ Crystals," Materials Chemistry and Physics, Vol. 83, 2004, pp. 158-168.

[23] M. Barsoum, "Fundamentals of Ceramics," McGraw-Hill, New York, 1997

[24] H. E. Atyia, "Deposition Temperature Effect on the Electric and Dielectric Properties of $\mathrm{InSbSe}_{3}$ Thin Films," Vacuum, Vol. 81, 2007, pp. 590-598. doi:10.1016/j.vacuum.2006.07.011

[25] L. L. Hench and J. K. West, "Principles of Electronic Ceramics," Wiley, New York, 1990.

[26] R. H. Chen, R. J. Wang, T. M. Chen and C. S. Shern, "Studies on the Dielectric Properties and Structural Phase Transition of $\mathrm{K}_{2} \mathrm{SO}_{4}$ Crystal," Journal of Physics and Chemistry of Solids, Vol. 61, 2000, pp. 519-527. doi:10.1016/S0022-3697(99)00246-2

[27] L. Sirdeshmukh, K. Krishna Kumar, S. B. Laxaman, A. R. Krishna and G. Sathaiah, "Dielectric Properties and Electrical Conduction in Yttrium Iron Garnet (YIG)," Bulletin of Materials Science, Vol. 21, 1998, p. 219. doi:10.1007/BF02744973

[28] R. P. Mahajan, K. K. Patankar, M. B. Kothale and S. A. Patil, "Conductivity, Dielectric Behaviour and MagnetoElectric Effect in Copper Ferrite-Barium Titanate Composites," Bulletin of Materials Science, Vol. 23, 2000, p. 273. doi:10.1007/BF02720082

[29] J. Appel, "Polarons," Solid State Physics, Vol. 21, 1968, p. 193. doi:10.1016/S0081-1947(08)60741-9

[30] K. Amarendra Singh, T. C. Goel and R. G. Mendiratta, "Dielectric Properties of Mn-substituted Ni-Zn Ferrites," Journal of Applied Physics, Vol. 91, No. 10, 2002, pp. 6626-6629.

[31] V. Gupta, K. K. Bamzai, P. N. Kotru and B. M. Wanklyn, "Dielectric Properties, AC Conductivity and Thermal Behaviour of Flux Grown Cadmiumtitanate Crystals,"
Materials Science and Engineering B, Vol. 130, 2006, pp. 163-172.

[32] M. M. El-Desoky, "Dielectric Behaviour and AC Conductivity of Sodium Borate Glass Containing CoO," Journal of Physics and Chemistry of Solids, Vol. 59, No. 9, 1998, pp. 1659-1666.

[33] F. Salman, S. Aboelhssan, E. Sheha and M. K. Elmansy, "Dielectric Properties and Conductivity of $\mathrm{KHCO}_{3}$," Turkish Journal of Physics, Vol. 28, 2004, pp. 57-63.

[34] D. Petrova, S. Dobreva, M. Veleva. J. Macicek and M. Gospodinov, "Growth, Structure, Dielectric Behavior and AC-Conductivity of Pyrochlore Lead-Scandium Tantalate Single Crystals," Material Research Bulletin, Vol. 32, No. 11, 1997, pp. 1543-1549. doi:10.1016/S0025-5408(97)00136-0

[35] P. Suryanarayana, H. N. Acharya and K. Rao, "Dielectric Properties of Mercuric Iodide $\left(\mathrm{HgI}_{2}\right)$ Single Crystals," Journal of Materials Science Letters, Vol. 3, 1984, p. 21. doi:10.1007/BF00720065

[36] P. Smyth, "Dielectric Behaviour and Structure," McGrawHill, New York, 1965.

[37] A. J. Moulson and J. M. Herbert, "Electroceramics: Materials Properties Applications," Chapman \& Hall, New York, 1990.

[38] S. Bhat, S. K. Khosa, P. N. Kotru and R. P. Tandon, "Dielectric Studies of Lanthanum Heptamolybdate Crystals Grown from Gels," Materials Science and Engineering B, Vol. 30, 1995, p. 7. doi:10.1016/0921-5107(94)01129-X

[39] S. Bhat, S. K. Khosa, P. N. Kotru and R. P. Tandon, "Dielectric Characteristics of Neodymium Heptamolybdate Crystals Grown by Gel Encapsulation Technique," Crystal Research and Technology, Vol. 30, No. 2, 1995, pp. 267-273. doi:10.1002/crat.2170300225

[40] S. Bhat, S. K. Khosa, P. N. Kotru and R. P. Tandon, Tandon, "Dielectric Characteristics of Gel-Grown Mixed Neodymium-Lanthanum-Heptamolybdate Crystals," Journal of Materials Science Letters, Vol. 14, 1995, p. 564. doi:10.1007/BF00275377

[41] Balarew and R. Duhlew, "Application of the Hard and Soft Acids and Bases Concept to Explain Ligand Coordination in Double Salt Structures," Journal of Solid State Chemistry, Vol. 55, 1984, p. 1. doi:10.1016/0022-4596(84)90240-8

[42] A. K. Jonscher, "The 'Universal' Dielectric Response," Nature, Vol. 267, 1977, p. 673.

[43] K. Jonscher, "Analysis of the Alternating Current Properties of Ionic Conductors," Journal of Materials Science, Vol. 13, 1978, p. 553. doi:10.1007/BF00541805

[44] M. D. Ingram, "Ionic Conductivity in Glass," Physics and Chemistry of Glasses, Vol. 28, 1987, p. 215.

[45] A. Angell, "Dynamic Processes in Ionic Glasses," Chemical Reviews, Vol. 90, 1990, p. 523. doi:10.1021/cr00101a006

[46] A. R. Long, "Frequency-Dependent Loss in Amorphous Semiconductors," Advances in Physics, Vol. 31, 1982, p. 553. doi:10.1080/00018738200101418 
[47] S. R. Elliott, "A.C. Conduction in Amorphous Chalcogenide and Pnictide Semiconductors," Advances in Physics, Vol. 36, 1987, p. 135. doi:10.1080/00018738700101971

[48] M. Pollak and T. H. Geballe, "Low-Frequency Conductivity Due to Hopping Processes in Silicon," Physical Review, Vol. 122, 1961, p. 1742. doi:10.1103/PhysRev.122.1742

[49] P. G. Bruce, R. A. West and D. P. Almond, "A New Analysis of AC Conductivity Data in Single Crystal ß-Alumina," Solid State Ionics, Vol. 7, 1982, p. 57. doi:10.1016/0167-2738(82)90069-8

[50] W. K. Lee, J. F. Liu and A. S. Nowick, "Limiting Behavior of AC Conductivity in Ionically Conducting Crystals and Glasses: A New Universality," Physical Review Letters, Vol. 67, 1991, p. 1559. doi:10.1103/PhysRevLett.67.1559

[51] L. Murowski and R. J. Barczynski, "Dielectric Properties of Transition Metal Oxide Glasses," Journal of NonCrystalline Solids, Vol. 185, 1995, p. 84. doi:10.1016/0022-3093(95)00677-X
[52] J. W. Verwey and Heilman, "Physical Properties and Cation Arrangement of Oxides with Spinel Structures I. Cation Arrangement in Spinels," Journal of Chemical Physics, Vol. 15, 1947, p. 174.

[53] N. Chand and D. Jain, "Evaluation of A.C. Conductivity Behaviour of Graphite Filled Polysulphide Modified Epoxy Composites," Bulletin of Materials Science, Vol. 27, 2004, p. 227. doi:10.1007/BF02708511

[54] C. A. Angell, "Mobile Ions in Amorphous Solids," Annual Review of Physical Chemistry, Vol. 43, 1992, p. 693. doi:10.1146/annurev.pc.43.100192.003401

[55] A. MA, E. A. E. Wahhab, A. E. Bekheet and H. E. Atyia, "Effect of Annealing on the AC Conductivity and the Dielectric Properties of $\mathrm{In}_{2} \mathrm{Te}_{3}$ Thin Films," Acta Phys Polon A, Vol. 28, 2000, p. 403.

[56] S. S. Fouad, A. E. Bekheet and A. M. Farid, "Derivation of a Relation between the Conduction Mechanism and Chemical Bonding of Amorphous $\mathrm{Ge}_{15} \mathrm{Se}_{85-\mathrm{x}} \mathrm{Ag}_{\mathrm{x}}$ Alloys," Physica B, Vol. 163, 2002, p. 3229. 